\title{
On the $\alpha$-nonbacktracking centrality for complex networks: Existence and limit cases
}

\author{
Regino Criado ${ }^{\mathrm{a}, \mathrm{b}, \mathrm{c}}$, Julio Flores ${ }^{\mathrm{a}, \mathrm{b}, *}$, Esther García ${ }^{\mathrm{a}}$, \\ Alejandro J. García del Amo ${ }^{\mathrm{a}, \mathrm{b}}$, Ángel Pérez ${ }^{\mathrm{a}}$, Miguel Romance $\mathrm{ab, \textrm {c }}$ \\ a Departamento de Matemática Aplicada, Ciencia e Ingeniería de los Materiales y Tecnología Elctrónica, ESCET Universidad Rey Juan \\ Carlos, C/Tulipán s/n, 28933 Móstoles (Madrid), Spain \\ b Center for Computational Simulation, Universidad Politécnica de Madrid, 28223 Pozuelo de Alarcón (Madrid), Spain \\ ${ }^{\mathrm{c}}$ Data, Networks and Cybersecurity Research Institute, Univ. Rey Juan Carlos, 28028 Madrid, Spain
}

\section{A R T I C L E I N F O}

\section{Article history:}

Received 31 January 2018

Received in revised form 20 September 2018

\section{Keywords:}

Nonbacktracking centrality

Alpha-centrality

Perturbative analysis of matrices

Spectral analysis of complex networks

\begin{abstract}
A B S T R A C T
Nonbactracking centrality was introduced as an attempt to correct some deficiencies of eigenvector centrality. In this work the $\alpha$-nonbacktracking centrality is introduced as an extension that interpolates between the nonbacktracking centrality of the edges of a directed network and the eigenvector centrality of the corresponding directed line graph. The existence of this new $\alpha$-nonbacktracking centrality is proved in terms of the connectivity of the original network.

We prove that the limit of the $\alpha$-nonbacktracking centrality when $\alpha$ decreases to zero exists and is well defined. Moreover, it coincides with the nonbacktracking centrality when this measure is defined. With the same techniques we also prove the convergence of PageRank vectors to the eigenvector centrality vector when the damping factor tends to 1 .
\end{abstract} (C) 2018 Elsevier B.V. All rights reserved.

\section{Introduction}

Complex networks have been used with great success to model many real-world systems in fields ranging from biology (which include issues such as metabolic pathways, protein folding or genetic regulatory networks) to the Internet, the World Wide Web and other technological systems [1-8]. Research on these issues must necessarily encompass a diversity of views that include different complementary aspects of the network structure, and the huge complexity of these objects requires new tools coming from other fields, including matrix analysis, statistical mechanics and computer sciences [9,1,10,2,3,11]. So, complex networks have recently attracted the attention of numerous research areas and consequently the mathematical and computational study of complex networks has experienced very significant growth in recent years $[2,4,8]$.

The spectral properties of the adjacency matrix provide great insight into the structure and function of complex networks. Specifically, the spectral radius and its associated principal eigenvector are fundamental in the understanding of nodes' centrality. Eigenvector centrality [12] takes into account that not all the edges or relations are equal, since a node is more important (or influential) if the nodes with which it is connected are, in turn, important or influential nodes. Eigenvector centrality has been analyzed and extended for use in different contexts and applications [2,13-16,11]. Specifically, in [14]

\footnotetext{
* Corresponding author at: Departamento de Matemática Aplicada, Ciencia e Ingeniería de los Materiales y Tecnología Elctrónica, ESCET Universidad Rey Juan Carlos, C/Tulipán s/n, 28933 Móstoles (Madrid), Spain.

E-mail addresses: regino.criado@urjc.es (R. Criado),julio.flores@urjc.es (J. Flores), esther.garcia@urjc.es (E. García), alejandro.garciadelamo@urjc.es (A.J.G. del Amo), angel.perez@urjc.es (Á. Pérez), miguel.romance@urjc.es (M. Romance).
} 
the authors very rightly observe that eigenvector centrality in a network can be artificially high on nodes with high degree (hubs). The reason is simple and can be easily grasped in the following sentence:

I am important, then my friends become important, then I become even more important, then. .

Thus the pattern "My importance depends on my friend's importance" tends to give priority to hubs just for their own nature, i.e., a hub with an elevated eigenvector centrality transmits it to its neighbors, who in turn reflect it back and inflate the hub's centrality artificially. Therefore, if we can avoid this feedback centrality will behave much more realistically. Thus, in [14] an attempt to correct this weakness is proposed by using the nonbacktracking centrality. The idea is to use a modified eigenvector centrality that is similar in many ways but with an important change: to calculate the centrality of a particular node, the authors consider the centrality of its neighbors, in a similar way as is done with the usual centrality eigenvector, but the centrality of its neighbors is now calculated in the absence of that particular node. This centrality measure can be calculated using the Hashimoto or nonbacktracking matrix [17,18], as pointed out by the authors in [14]. As we will see in the next section, this matrix is closely related to the adjacency matrix of the line graph corresponding to the network under consideration. In any case, it is important to highlight that when the authors in [14] introduced the nonbacktracking centrality vector a natural objection arises: can we be sure that such a vector exists? More precisely, Perron-Frobenius theorem [19] guarantees that a non-negative and irreducible square matrix $A$ has a non-negative eigenvector associated to its spectral radius $\rho(A)$. But if $A$ were not irreducible then Perron-Frobenius theorem can no longer be used and hence the unicity of eigenvector gets compromised; however it is this unicity which allows us to speak of the nonbacktracking centrality eigenvector.

The main goal of this work is to define a new centrality measure, the $\alpha$-nonbacktracking centrality, that makes possible to solve the problems related to the uniqueness of nonbactracking principal eigenvector. We prove that the $\alpha$-nonbacktracking centrality converges to a unique vector when $\alpha \rightarrow 0^{+}$and this vector coincides with the nonbacktracking centrality vector when this last one is unique. Moreover, the same techniques demonstrate the convergence of PageRank vectors when the damping factor tends to 1 , which gives an idea of the applicability of our result. Moreover our new centrality measure provides a unified model that interpolates between nonbacktracking centrality of the network edges and eigenvector centrality of the line graph.

The structure of the paper is as follows. After this introduction, Section 2 is devoted to introduce and recall some preliminary results and definitions. In Section 3 the $\alpha$-nonbacktracking centrality is introduced in order to avoid the problem of irreducibility and lack of uniqueness of the nonbacktracking principal eigenvector. Finally, in Section 4 a result related to the convergence of the spectral radii and the Perron vectors of a family of irreducible non-negative matrices is obtained and, moreover, we point out how our result can be used to prove the convergence of the PageRank vectors when the damping factor $q \rightarrow 1^{-}$.

\section{Notation and preliminaries}

Let $G=(V, E)$ be a directed network with no loops, where a loop means an edge from a node to itself. Denote by $i \rightarrow j$ the edge from $i$ to $j$. Let us denote by $A=\left(a_{i j}\right)$ the adjacency matrix of $G$, where

$$
a_{i j}= \begin{cases}1 & \text { if there is an edge } i \rightarrow j \\ 0 & \text { otherwise. }\end{cases}
$$

For the case of a non directed network we will consider the associated directed network in which each edge $\{i, j\}$ is replaced by the two directed edges $i \rightarrow j$ and $j \rightarrow i$. Notice that the adjacency matrix of a non directed network coincides with the adjacency matrix of the associated directed network.

In order to remove feedback the heuristics goes as follows: the centrality of edge $k \rightarrow l$ is proportional to the sum of the centralities of all edges incident on $k \rightarrow l$ except edge $l \rightarrow k$. Here $i \rightarrow j$ is incident on $k \rightarrow l$ if $j=k$.

The heuristics is revealed in the Hashimoto matrix [17] as follows: We start fixing an order in $E$ (for instance the lexicographic order), then we take the adjacency matrix for edge incidence

$$
(B(0))_{i \rightarrow j, k \rightarrow l}=\left\{\begin{array}{cc}
1 & j=k \text { and } i \neq l, \\
0 & \text { otherwise, }
\end{array}\right.
$$

that is,

$$
(B(0))_{i \rightarrow j, k \rightarrow l}=\delta_{j k}\left(1-\delta_{i l}\right),
$$

where $\delta_{i j}$ is the Kronecker's delta, i.e.,

$$
\delta_{i j}=\left\{\begin{array}{cc}
1 & i=j \\
0 & \text { otherwise. }
\end{array}\right.
$$

Notice that the Hashimoto matrix is closely related to the adjacency matrix of $L(G)$, the line graph of $G$

$$
\left(M_{L(G)}\right)_{i \rightarrow j, k \rightarrow l}=\delta_{j k}=\left\{\begin{array}{cc}
1 & j=k, \\
0 & \text { otherwise, }
\end{array}\right.
$$

Recall that $i \rightarrow j$ is incident on $k \rightarrow l$ if $j=k$. 


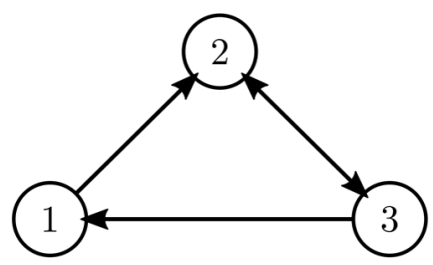

Fig. 1. An example of a directed network $G$ with 3 nodes.

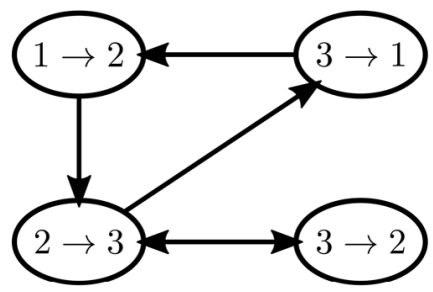

Fig. 2. The line graph $L(G)$.

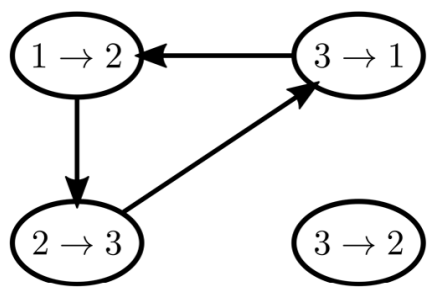

Fig. 3. The network associated to $B(0)$.

Example 2.1. Consider the network $G=(V, E)$ of three nodes represented in Fig. 1.

In this case, by taking the lexicographic order on the edges of $G$, i.e. the ordering $\{1 \rightarrow 2,2 \rightarrow 3,3 \rightarrow 1,3 \rightarrow 2\}$, we get the following matrices:

$$
B(0)=\left(\begin{array}{llll}
0 & 1 & 0 & 0 \\
0 & 0 & 1 & 0 \\
1 & 0 & 0 & 0 \\
0 & 0 & 0 & 0
\end{array}\right), \quad M_{L(G)}=\left(\begin{array}{cccc}
0 & 1 & 0 & 0 \\
0 & 0 & 1 & 1 \\
1 & 0 & 0 & 0 \\
0 & 1 & 0 & 0
\end{array}\right) .
$$

Notice that the Hashimoto matrix is $B(0)$ and is part of the adjacency matrix $M_{L(G)}$ of the line graph of $G=(V, E)$.

The relation between $B(0)$, the adjacency matrix of $L(G)$ and their respective associated networks is illustrated in Fig. 2 and Fig. 3.

Remark 2.2. Note that $\rho(B(0)) \leq \rho(L(G))$ since $B(0) \leq M_{L(G)}$, where the notation $\leq$ for matrices means that each entry of the matrix on the left is less than or equal to the corresponding coefficient of the matrix on the right. Similarly, we say that a vector $\mathbf{v}>0$ if all its entries are positive.

It is important to highlight the existence of strong relationships between the eigenvector centrality of a given network $G$, the eigenvector centrality of its line graph $L(G)$ and the eigenvector centrality of the bipartite network $B(G)$ associated to $G$, both in the case of $G$ being a non directed network [20] and in the case of $G$ being a directed network [21].

Thus the nonbacktracking centrality of edges is a function $\mathbf{c}(0): E \rightarrow[0,1]$ that satisfies:

- $\sum_{(k \rightarrow l) \in E} c(0)_{k \rightarrow l}=1$ (normalization).

- $c(0)_{k \rightarrow l}$ is proportional to the sum of $c(0)_{j \rightarrow k}$ where $j=l$ is disregarded. 


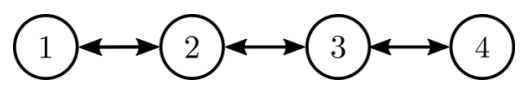

Fig. 4. An example network $G$ with 4 nodes.

In terms of $B(0)$

$$
c(0)_{i \rightarrow j}=\frac{1}{\lambda} \sum_{(k \rightarrow l) \in E}(B(0))_{k \rightarrow l, i \rightarrow j} c(0)_{k \rightarrow l} .
$$

Thus, if $\mathbf{c}(0)=\left(c(0)_{i \rightarrow j}\right)_{(i \rightarrow j) \in E}$ is a column vector, then $\lambda \mathbf{c}(0)=B(0)^{t} \mathbf{c}(0)$.

We remark that the nonbacktracking centrality of each edge in $E$ is ranked by means of a normalized non-negative eigenvector of $B(0)^{t}$.

At this point, once the nonbacktracking centrality of edges has been defined, it is possible to define the nonbacktracking centrality of nodes in the following natural way:

Definition 2.3 ([14]). The nonbacktracking centrality of node $i$ is the sum of centralities of all edges starting on $i$, in other words, the sum of centralities of all edges $i \rightarrow k$.

Remark 2.4. As pointed out in Section 1, if $B(0)$ is not irreducible, Perron-Frobenius theorem cannot be used to ensure the unicity of the nonbacktracking centrality eigenvector of edges and of nodes. For instance if we consider the network depicted if Fig. 4, and we choose the lexicographical order on the edges, the spectral radius of $B(0)^{t}$ equals 0 and the corresponding eigenspace is $\{(0, a, 0,0, b, 0)\}_{a, b \in \mathbb{R}}$. Note that any vector of the form $(0, a, 0,0,1-a, 0)$, with $a \in[0,1]$, fits the definition of nonbacktracking edge centrality. As a consequence, the nonbacktracking centrality of nodes 1 and 4 equals 0 , and the nonbacktracking centralities of nodes 2 and 3 are $a$ and $1-a$ respectively, for any choice of $a \in[0,1]$.

\section{3. $\alpha$-nonbacktracking centrality: existence and computation}

In this section the $\alpha$-nonbacktracking centrality is introduced. The objective is twofold: on the one hand we want to avoid the irreducibility problem that results in lack of unicity for the nonbacktracking eigenvector; on the other hand we are going to provide a unified model that interpolates between nonbacktracking centrality and eigenvector centrality.

Now we present the heuristics that support a definition consistent with our goal. Given an order in $E$ and $\alpha \in[0,1]$, the edge $\alpha$-nonbacktracking centrality $\mathbf{c}(\alpha): E \rightarrow[0,1]$ should satisfy:

- $\sum_{(k \rightarrow l) \in E} c(\alpha)_{k \rightarrow l}=1$ (normalization),

- $c(\alpha)_{k \rightarrow l}$ is proportional to the sum of $c(\alpha)_{j \rightarrow k}$, where $j \rightarrow k$ is incident on $k \rightarrow l$ and the case $j=l$ is admissible although dampened by $\alpha$.

Thus, the edge incidence information is collected in the following edge adjacency matrix

$$
\begin{aligned}
& (B(\alpha))_{i \rightarrow j, k \rightarrow l}=\left\{\begin{array}{cc}
1 & j=k \text { and } i \neq l, \\
\alpha & j=k \text { and } i=l, \\
0 & \text { otherwise, }
\end{array}\right. \\
& (B(\alpha))_{i \rightarrow j, k \rightarrow l}=\delta_{j k}\left(1+(\alpha-1) \delta_{i l}\right) .
\end{aligned}
$$

Remark 3.1. For $\alpha=0$ we get the Hashimoto matrix $B(0)$, while for $\alpha=1$ the matrix of $L(G)$ is recovered. Therefore, the nonbacktracking centrality and the eigenvector centrality of $L(G)$ are recovered for $\alpha=0$ and $\alpha=1$. Note that the eigenvector centrality of $L(G)$ is strongly related to the eigenvector centrality of $G[20,21]$.

Theorem 3.2. Let $G=(V, E)$ be a directed network with no isolated nodes. Then, $G$ is strongly connected if and only if $B(\alpha)$ is irreducible for $\alpha \in(0,1]$.

Proof. It is well known that $G$ is strongly connected if and only if $L(G)$ is strongly connected (cf. [22], p. 44), or equivalently $B(1)$ is irreducible. Similarly, $G$ is strongly connected if and only if $B(\alpha)$ is irreducible for all $\alpha \in(0,1]$.

Note that we cannot expect Theorem 3.2 to be extended to $\alpha=0$, as the network presented in Example 2.1 shows. In that case $G$ is strongly connected but $B(0)$ is not irreducible since $3 \rightarrow 2$ is isolated. Despite this inconvenience, there are some relationships between the irreducibility of matrix $B(0)$ and structural properties of the original graph $G$, as we will show. First, the following result shows that the strongly connectivity of $G$ is a necessary condition for the irreducibility of $B(0)$. 
Theorem 3.3. Let $G=(V, E)$ be a directed network with $n \geq 3$ nodes and no isolated nodes. If $B(0)$ is irreducible then $G$ is strongly connected.

Proof. It is easy to check that since $G=(V, E)$ has no isolated nodes and $B(0)$ is irreducible, then for every node $i \in V$ either the in-degree and the out-degree must be greater than zero. Hence for every node $i$, there exist $j_{1}, j_{2} \in V\left(\operatorname{possibly} j_{1}=j_{2}\right)$ such that $\left(i \rightarrow j_{1}\right),\left(j_{2} \rightarrow i\right) \in E$. Hence if we take $i, k \in V$ we can always construct a path from $i$ to $k$ simply by taking a path (that exists, since $B(0)$ is irreducible) from $i \rightarrow j_{1}$ to $j_{2} \rightarrow k$, and therefore $G$ is strongly connected.

If we look for sufficient conditions for the irreducibility of $B(0)$, we need to add some extra structural properties to the strongly connectivity of $G$ as the following result shows:

Theorem 3.4. Let $G=(V, E)$ be a non directed network with $n \geq 2$ nodes, then

(i) If $G$ is connected and for every edge $\{i, j\} \in E$ there are at least two cycles in $G$ that contain $\{i, j\}$ (disjoints apart from $\{i, j\}$ ), then $B(0)$ is irreducible.

(ii) If there is a node $i \in V$ with degree 1 , then $B(0)$ is not irreducible.

Proof. (i) Since $G$ is connected, the irreducibility of $B(0)$ is obtained if for every $\{i, j\} \in E$ we can find a path from $i \rightarrow j$ to $j \rightarrow i$, but this is straightforward from the fact that there are two disjoint cycles that contain $\{i, j\}$.

(ii) If we take $i \in V$ with degree 1 , there is a unique $j \in V$ such that $\{i, j\} \in E$. In this case, $j \rightarrow i$ is isolated in $B(0)$ and therefore it is not irreducible.

In the following, we will assume that $G$ is a strongly connected network and $\alpha \in(0,1]$. We define the edge $\alpha$ nonbacktracking centrality

$$
\mathbf{c}(\alpha)=\left(c(\alpha)_{i \rightarrow j}\right)_{(i \rightarrow j) \in E}
$$

as the positive normalized eigenvector associated to the spectral radius of $B(\alpha)$.

Definition 3.5. Let $\alpha \in(0,1]$. The $\alpha$-nonbacktracking centrality $c(\alpha)_{i}$ of node $i$ is the sum of centralities $c(\alpha)_{i \rightarrow k}$ of all the edges $i \rightarrow k$ (edges starting on $i$ ), i.e.,

$$
c(\alpha)_{i}=\sum_{i \rightarrow k} c(\alpha)_{i \rightarrow k} .
$$

Remark 3.6. In the next section, we will prove that $\lim _{\alpha \rightarrow 0^{+}} \mathbf{c}(\alpha)$ exists. Call this limit $\mathbf{c}$. Evidently $\mathbf{c}$ is non-negative and has norm one. Also $\mathbf{c}$ is easily seen to belong to the eigenspace associated to the spectral radius $\rho(B(0))$. Note that, due to the possible lack of irreducibility of $B(0)$, such eigenspace may have dimension greater than 1 ; hence choosing one centrality eigenvector in this eigenspace is certainly ambiguous. In this context $\mathbf{c}$ clearly appears as a natural candidate as the nonbacktracking centrality eigenvector of $B(0)$.

Definition 3.7. Let $G=(V, E)$ be a directed network with $n$ nodes and $m$ edges, and fix an order in $E$. For a given $\mathbf{v}=\left(v_{i \rightarrow j}\right)_{(i \rightarrow j) \in E} \in \mathbb{R}^{m}$ and a given node $i \in V$ we denote

$$
v_{i}^{\text {out }}=\sum_{j \in N(i)} v_{i \rightarrow j}=\sum_{j=1}^{n} a_{i j} v_{i \rightarrow j}, \quad v_{i}^{\text {in }}=\sum_{j \in N(i)} v_{j \rightarrow i}=\sum_{j=1}^{n} a_{j i} v_{j \rightarrow i},
$$

where $N(i)$ denotes the set of neighbors of node $i, A=\left(a_{i j}\right)$ is the adjacency matrix of $G$, and we assume $v_{i \rightarrow j}=0$ if $(i \rightarrow j) \notin E$. Now we define

$$
\mathbf{v}^{\text {out }}=\left(v_{i}^{\text {out }}\right)_{i \in V}, \mathbf{v}^{\text {in }}=\left(v_{i}^{\text {in }}\right)_{i \in V} .
$$

For example, consider the network depicted in Fig. 4 and fix the lexicographical order on the edges. Choose for instance the vector $\mathbf{v}=(1,2,3,3,5,6) \in \mathbb{R}^{6}$ and take node number 3 . Then

$$
\begin{aligned}
& v_{3}^{\text {out }}=\sum_{j \in N(3)} v_{3 \rightarrow j}=v_{3 \rightarrow 2}+v_{3 \rightarrow 4}=3+5=8, \\
& v_{3}^{\text {in }}=\sum_{j \in N(3)} v_{j \rightarrow 3}=v_{2 \rightarrow 3}+v_{4 \rightarrow 3}=3+6=9 .
\end{aligned}
$$

Proceeding similarly for the rest of the nodes we finally get

$$
\mathbf{v}^{\text {out }}=(1,5,8,6) \text { and } \mathbf{v}^{\text {in }}=(2,4,9,5) \text {. }
$$

Notice that, with this notation,

$$
c(\alpha)_{i}=\sum_{j \in N(i)} c(\alpha)_{i \rightarrow j}=\sum_{j=1}^{n} a_{i j} c(\alpha)_{i \rightarrow j}=c(\alpha)_{i}^{\text {out }} .
$$


Note that one of the main drawbacks of the nonbacktracking centrality is the fact that it needs the computation of eigenvectors of big size matrices. In the particular case of non directed graphs we are going to present a result that allows to obtain $\rho(B(\alpha))$ and $\mathbf{c}(\alpha)$ by using a block matrix smaller than $B(\alpha)$ with techniques similar to those in [18]. Recall that a directed network can always be associated to a non directed one by replacing each edge $\{i, j\}$ by the two directed edges $i \rightarrow j$ and $j \rightarrow i$.

Theorem 3.8. Let $G$ be a non directed network with no loops, let $(V, E)$ be its associated directed network with $n$ nodes and $m$ edges, and let $\alpha \in(0,1]$. If $\mathbf{v} \in \mathbb{R}^{m}$ is an eigenvector of $B(\alpha)^{t}$, with eigenvalue $\lambda$, and either $\mathbf{v}^{\text {out }} \neq 0$ or $\mathbf{v}^{\text {in }} \neq 0$, then $\left(\begin{array}{c}\mathbf{v}^{\text {out }} \\ \mathbf{v}^{\text {in }}\end{array}\right) \in \mathbb{R}^{2 n}$ is an eigenvector (with the same eigenvalue $\lambda$ ) of matrix $\tilde{B}(\alpha)$ given by

$$
\tilde{B}(\alpha)=\left(\begin{array}{cc}
0 & D+(\alpha-1) I \\
(\alpha-1) I & A
\end{array}\right),
$$

where $D$ is the diagonal matrix in which elements are the degrees $d(i)$, A denotes the adjacency matrix of $G$, and $I$ is the identity matrix of size $n$. In particular, if $\mathbf{v}>0$ is an eigenvector corresponding to the spectral radius $\rho(B(\alpha))$, then

$$
\rho(B(\alpha))\left(\begin{array}{c}
\mathbf{v}^{\text {out }} \\
\mathbf{v}^{\text {in }}
\end{array}\right)=\tilde{B}(\alpha)\left(\begin{array}{c}
\mathbf{v}^{\text {out }} \\
\mathbf{v}^{\text {in }}
\end{array}\right) .
$$

Proof. Let $\mathbf{v} \in \mathbb{R}^{m}$ and $(k \rightarrow l) \in E$. Then

$$
\begin{aligned}
\left(B(\alpha)^{t} \mathbf{v}\right)_{k \rightarrow l} & =\sum_{(x \rightarrow y) \in E} B(\alpha)_{k \rightarrow l, x \rightarrow y}^{t} v_{x \rightarrow y}=\sum_{(x \rightarrow y) \in E} B(\alpha)_{x \rightarrow y, k \rightarrow l} v_{x \rightarrow y} \\
& =\sum_{x, y=1}^{n} \delta_{y k}\left(1+(\alpha-1) \delta_{x l}\right) a_{x y} v_{x \rightarrow y}=\alpha a_{l k} v_{l \rightarrow k}+\sum_{x \neq l} a_{x k} v_{x \rightarrow k} \\
& =\left(\sum_{x=1}^{n} a_{x k} v_{x \rightarrow k}\right)+(\alpha-1) a_{l k} v_{l \rightarrow k} .
\end{aligned}
$$

Therefore, for $i \in V$

$$
\begin{aligned}
\left(B(\alpha)^{t} \mathbf{v}\right)_{i}^{\text {out }} & =\sum_{j=1}^{n} a_{i j}\left(B(\alpha)^{t} \mathbf{v}\right)_{i \rightarrow j} \\
& =\sum_{j=1}^{n} a_{i j}\left(\left(\sum_{x=1}^{n} a_{x i} v_{x \rightarrow i}\right)+(\alpha-1) a_{j i} v_{j \rightarrow i}\right) \\
& =\left(\sum_{j \in N(i)} \sum_{x \in N(i)} v_{x \rightarrow i}\right)+(\alpha-1) \sum_{j \in N(i)} v_{j \rightarrow i} \\
& =|N(i)| v_{i}^{i n}+(\alpha-1) v_{i}^{i n}=(d(i)+(\alpha-1)) v_{i}^{i n},
\end{aligned}
$$

where $d(i)$ is the degree of node $i$. Similarly, for $i \in V$

$$
\begin{aligned}
\left(B(\alpha)^{t} \mathbf{v}\right)_{i}^{\text {in }} & =\sum_{j=1}^{n} a_{j i}\left(B(\alpha)^{t} \mathbf{v}\right)_{j \rightarrow i} \\
& =\sum_{j=1}^{n} a_{j i}\left(\left(\sum_{x=1}^{n} a_{x j} v_{x \rightarrow j}\right)+(\alpha-1) a_{i j} v_{i \rightarrow j}\right) \\
& =\left(\sum_{j \in N(i)} \sum_{x \in N(j)} v_{x \rightarrow j}\right)+(\alpha-1) \sum_{j \in N(i)} v_{i \rightarrow j} \\
& =\sum_{j=1}^{n} a_{j i} v_{j}^{\text {in }}+(\alpha-1) v_{i}^{\text {out }} .
\end{aligned}
$$

Now, we get that

$$
\left(\begin{array}{c}
\left(B(\alpha)^{t} \mathbf{v}\right)^{\text {out }} \\
\left(B(\alpha)^{t} \mathbf{v}\right)^{\text {in }}
\end{array}\right)=\tilde{B}(\alpha)\left(\begin{array}{c}
\mathbf{v}^{\text {out }} \\
\mathbf{v}^{\text {in }}
\end{array}\right) .
$$


Then, if $\mathbf{v} \in \mathbb{R}^{m}$ is an eigenvector of $B(\alpha)^{t}$, with eigenvalue $\lambda$, we have

$$
\left(B(\alpha)^{t} \mathbf{v}\right)_{i}^{\text {out }}=\sum_{j=1}^{n} a_{i j}\left(B(\alpha)^{t} \mathbf{v}\right)_{i \rightarrow j}=\lambda \sum_{j=1}^{n} a_{i j} v_{i \rightarrow j}=\lambda v_{i}^{\text {out }}
$$

and

$$
\left(B(\alpha)^{t} \mathbf{v}\right)_{i}^{\text {in }}=\lambda v_{i}^{\text {in }} .
$$

Therefore,

$$
\lambda\left(\begin{array}{c}
\mathbf{v}^{\text {out }} \\
\mathbf{v}^{\text {in }}
\end{array}\right)=\tilde{B}(\alpha)\left(\begin{array}{c}
\mathbf{v}^{\text {out }} \\
\mathbf{v}^{\text {in }}
\end{array}\right) .
$$

Remark 3.9. Note that if we take $\alpha=0$ in the last theorem we get the localization result for the nonbacktracking centrality presented in [14] while if $\alpha=1$, then

$$
\tilde{B}(1)=\left(\begin{array}{ll}
0 & D \\
0 & A
\end{array}\right) .
$$

In this case $\sigma(\tilde{B}(\alpha))=\sigma(A) \cup\{0\}$, and therefore any positive $\tilde{B}(1)$-eigenvector cannot have 0 as eigenvalue, which makes the 1-nonbacktracking centrality of nodes coincide with the eigenvector centrality of $G$.

\section{Limit case of $\alpha$-nonbacktracking centrality and other spectral centrality measures}

In this section we will study the convergence when $\alpha \rightarrow 0^{+}$of the spectral radii and the Perron vectors of a family of irreducible non-negative matrices $M_{\alpha}$ of the form $M_{0}+\alpha M$. This is the case when $M_{0}=B(0)^{t}$ is the transpose of the Hashimoto matrix perturbed by an $\alpha$-multiple of a matrix $M$, as proposed in Section 3. We will also point out how our result can be used to prove the convergence of the PageRank vectors when the damping factor tends to 1 .

Clearly $M_{0}+\alpha M$ converges to $M_{0}$ as $\alpha \rightarrow 0^{+}$and therefore it is easy to check that $\rho\left(M_{0}+\alpha M\right) \rightarrow \rho\left(M_{0}\right)$. This is well known and uses the fact that the composition of continuous functions is continuous. Indeed, the coefficients of a matrix depend continuously on the matrix (whatever the matrix norm considered) and therefore the characteristic polynomial depends continuously on the matrix. As the roots of the characteristic polynomial (over $\mathbb{C}$ ) continuously depend on its coefficients (see, for example [23]) and the absolute value is a continuous function we obtain, by composing all four previous dependencies, that the spectral radius varies continuously with the matrix.

Next we will justify that the family of Perron vectors of $M_{\alpha}$ converges as $\alpha \rightarrow 0^{+}$. The techniques used are known and make use of well established facts on analytical functions $[24,25]$.

Theorem 4.1. Let $M_{\alpha}=M_{0}+\alpha M, \alpha>0$, be a family of irreducible squared non-negative matrices of size $n$. Let $\mathbf{c}(\alpha)$ be the Perron vector of each $M_{\alpha}, \alpha>0$. Then the sequence of vectors $\{\mathbf{c}(\alpha)\}_{\alpha}$ converges to an eigenvector of $M_{0}$ when $\alpha \rightarrow 0^{+}$.

Proof. Let $\rho_{\alpha}$ be the spectral radius of $M_{\alpha}$ and let $\mathbf{c}(\alpha)=\left(c_{1}(\alpha), \ldots, c(\alpha)_{n}\right)$ be the Perron vector of $M_{\alpha}$. Let $A(\alpha):=M_{\alpha}-\rho_{\alpha} I$ where $I$ denotes the identity matrix of size $n$.

- Step 1: Let us express each $c(\alpha)_{j}, j=1, \ldots, n$, in terms of adjoints of elements of the matrix $A(\alpha)$. Since $M_{\alpha}$ is irreducible and $\rho_{\alpha}$ is the spectral radius, $\operatorname{rg}(A(\alpha))=n-1$. Let $\tilde{A}(\alpha)$ be the submatrix of $A(\alpha)$ containing the first $n-1$ rows. Without loss of generality we can assume that the rank of $\tilde{A}(\alpha)$ is $n-1$ and that the following linear map $F$ is bijective:

$$
\begin{aligned}
F: \mathbb{R}^{n} \longrightarrow \mathbb{R}^{n} \\
\left(\begin{array}{c}
x_{1} \\
\vdots \\
x_{n}
\end{array}\right) \longrightarrow\left(\begin{array}{c}
\left.\tilde{A}(\alpha)\left(\begin{array}{c}
x_{1} \\
\vdots \\
x_{n}
\end{array}\right)\right) . \\
x_{n}
\end{array}\right) .
\end{aligned}
$$

The matrix of this linear map with respect to the canonical basis will be denoted $F$ again. Notice that the matrix $F$ coincides with the matrix $A(\alpha)$ where the last row was replaced by $(0,0, \ldots, 0,1)$. Thus, the minors of the elements of the last row are the same for both matrices. Consequently $\operatorname{det}(F)=A(\alpha)_{n n}$, where $A(\alpha)_{n n}$ is the minor of the element $a_{n n}$ of the matrix $A(\alpha)$. In general, let us denote $A(\alpha)_{i j}$ the minor of the element $a_{i j}$ of $A(\alpha)$.

Since $F\left(\mathbf{c}(\alpha)^{t}\right)=\left(\overrightarrow{0}_{\mathbb{R}^{n-1}}, c(\alpha)_{n}\right)^{t}$ and $F$ is bijective,

$$
\mathbf{c}(\alpha)=F^{-1}\left(\begin{array}{l}
\overrightarrow{0}_{\mathbb{R}^{n-1}} \\
c(\alpha)_{n}
\end{array}\right)=c(\alpha)_{n}\left(F^{-1}\right)_{n},
$$


where $\left(F^{-1}\right)_{n}$ denotes the $n$th column of $F^{-1}$. From classic Cramer's rule we get that

$$
\begin{aligned}
\mathbf{c}(\alpha)=c(\alpha)_{n}\left(F^{-1}\right)_{n} & =\frac{c(\alpha)_{n}}{\operatorname{det}(F)}\left(\begin{array}{c}
(-1)^{n+1} A(\alpha)_{n 1} \\
(-1)^{n+2} A(\alpha)_{n 2} \\
\ldots \\
(-1)^{2 n} A(\alpha)_{n n}
\end{array}\right) \\
& =\frac{c(\alpha)_{n}}{A(\alpha)_{n n}}\left(\begin{array}{c}
(-1)^{n+1} A(\alpha)_{n 1} \\
(-1)^{n+2} A(\alpha)_{n 2} \\
\ldots \\
(-1)^{2 n} A(\alpha)_{n n}
\end{array}\right)
\end{aligned}
$$

and thus

$$
c(\alpha)_{j}=c(\alpha)_{n} \frac{(-1)^{n+j} A(\alpha)_{n j}}{A(\alpha)_{n n}}, \quad j=1, \ldots, n .
$$

Notice that $c(\alpha)_{j}>0$ implies $A(\alpha)_{n j} \neq 0$ for every $j$. Moreover, since $\mathbf{c}(\alpha)$ is the Perron vector,

$$
1=\|\mathbf{c}(\alpha)\|_{1}=\frac{c(\alpha)_{n}}{\left|A(\alpha)_{n n}\right|} \sum_{j}\left|A(\alpha)_{n j}\right|,
$$

which implies that $\left|A(\alpha)_{n n}\right|=c(\alpha)_{n} \sum_{j}\left|A(\alpha)_{n j}\right|$ and therefore

$$
c(\alpha)_{j}=\frac{\left|A(\alpha)_{n j}\right|}{\sum_{i}\left|A(\alpha)_{n i}\right|}=\frac{1}{1+\sum_{i \neq j} \frac{\left|A(\alpha)_{n i}\right|}{\left|A(\alpha)_{n j}\right|}} .
$$

Observe that each term $\frac{\left|A(\alpha)_{n i}\right|}{\left|A(\alpha)_{n j}\right|}$ in (4.2) is a quotient of polynomials in two variables $\alpha$ and $\rho_{\alpha}=\rho(\alpha)$.

- Step 2: Let us prove that for every $j \in\{1, \ldots, n\}$ and every $i \neq j$, the limit

$$
\lim _{\alpha \rightarrow 0^{+}} \frac{\left|A(\alpha)_{n i}\right|}{\left|A(\alpha)_{n j}\right|}
$$

always exists and belongs to $[0, \infty]$. Take the function $G(\alpha, \omega)=\operatorname{det}\left(M_{\alpha}-\omega I\right)$ defined on $\mathbb{C} \times \mathbb{C}$. Notice that $G(\alpha, \omega)$ is a polynomial of the two variables $\alpha, \omega$ of degree $n$. Hence

$$
G(\alpha, \omega)=\sum_{k=0}^{n} g_{k}(\alpha) \omega^{k},
$$

where $g_{k}(\alpha)$ is a polynomial in $\alpha$.

By choosing, for every $\alpha \in \mathbb{C}$, an element $\omega_{\alpha}$ in the spectrum $\sigma\left(M_{\alpha}\right)$ of $M_{\alpha}$ we have $G\left(\alpha, \omega_{\alpha}\right)=0$. Notice that for every $\alpha \in \mathbb{R}^{+} \cup\{0\}$ we can choose $\omega_{\alpha}=\rho_{\alpha}$, the spectral radius of $M_{\alpha}$ (by the Perron-Frobenius theorem for non-negative matrices and the continuity of the spectral radius observed above).

Thus, we have a function $\omega(\alpha)=\omega_{\alpha}$ defined on the complex plane satisfying $\omega(\alpha)=\rho_{\alpha}$ for $\alpha \in \mathbb{R}^{+} \cup\{0\}$ and such that $G\left(\alpha, \omega_{\alpha}\right)=0$ for all $\alpha \in \mathbb{C}$. According to section $\S 13$ in [25] such a function, $\omega(\alpha)$, is called an element of an algebraic function defined by $G(\alpha, \omega)=0$.

We can assume that equation $G(\alpha, \omega)=0$ is irreducible (see [25], Section $\S 13$ ). Observe that for a fixed $\alpha_{0}$ the equation $G\left(\alpha_{0}, \omega\right)=0$ will have, in general, $n$ distinct roots $\omega_{0}^{(i)}, i=1, \ldots, n$. As observed in [25], Section $\S 13$, there are two exceptions to this:

(i) $g_{n}\left(\alpha_{0}\right)=0$. Then the degree (in $\omega$ ) of the equation $G\left(\alpha_{0}, \omega\right)=0$ is lowered.

(ii) $G\left(\alpha_{0}, \omega\right)=0$ has multiple roots.

As noticed in Section $\S 13$ in [25], there are only finitely many values of $\alpha$, which we denote $a_{1}, \ldots, a_{r}$, for which either (i) or (ii) holds. These special values are called critical points and must therefore be isolated points. This implies that when the limit $\lim _{\alpha \rightarrow 0^{+}} \frac{\left|A(\alpha) n_{n i}\right|}{\left|A(\alpha)_{n j}\right|}$ is considered (here $\alpha \rightarrow 0^{+}$in the positive real axis), there must exist some $\delta>0$ such that every $\alpha \in(0, \delta)$ is a non-critical point, while $\alpha=0$ can be a critical point.

If $\alpha_{0}$ is not one of the critical points above then in Section $\S 14$ of [25] the equation $G(\alpha, \omega)=0$ is proved to define a single $n$-valued analytical function $\omega=F(\alpha)$. Equivalently, this equation defines $n$-branches of a multivalued analytical function. More importantly the critical points become poles of this function as proved in Section $\S 15$ of [25].

Since $\rho(\alpha)$ satisfies $G(\alpha, \rho(\alpha))=0$ for all $\alpha \in[0, \delta)$ it follows from the above that $\rho(\alpha)$ is an analytical function with 0 as a pole. But this certainly means that after applying L'Hospital's rule at most a finite number of times we get

$$
\lim _{\alpha \rightarrow 0^{+}} \frac{\left|A(\alpha)_{n i}\right|}{\left|A(\alpha)_{n j}\right|} \in[0, \infty]
$$




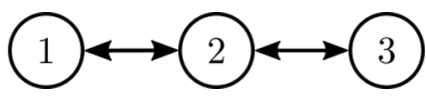

Fig. 5. An example network $G$ with 3 nodes.

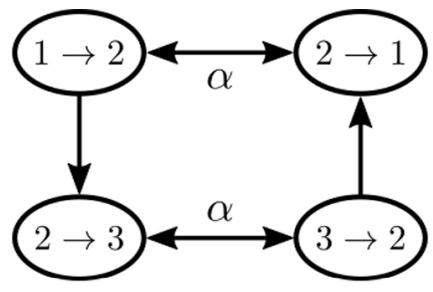

Fig. 6. The network associated to $B(\alpha)$.

- Step 3: The number of terms in the sum $\sum_{i \neq j} \mid \frac{A(\alpha)_{n i} \mid}{\left|A(\alpha)_{n j}\right|}$ is finite. Hence from Step 2 it follows that

$$
c(\alpha)_{j}=\frac{1}{1+\sum_{i \neq j} \frac{\left|A(\alpha)_{n i}\right|}{\left|A(\alpha)_{n j}\right|}}
$$

must converge and therefore the vector $\mathbf{c}(\alpha)=\left(c_{1}(\alpha), \ldots, c(\alpha)_{n}\right)$ must converge. Call this limit $\mathbf{c}$. Evidently $\mathbf{c}$ is non-negative and has norm one. Also $\mathbf{c}$ is easily seen to belong to the eigenspace associated to the spectral radius $\rho\left(M_{0}\right)$.

Remark 4.3. As was observed the vector $\mathbf{c} \equiv \lim _{\alpha} \mathbf{c}(\alpha)$ is non-negative, has norm one and belongs to the eigenspace associated to the spectral radius $\rho\left(M_{0}\right)$. This implies that in the case that the nonbacktracking centrality is well defined it must necessarily coincide with $\mathbf{c}$ as the eigenspace associated to the spectral radius has dimension 1 by Perron's theorem. This evidences that our definition coherently extends Newman et al.'s definition.

In addition Theorem 4.1 allows us to give a precise meaning to the nonbacktracking centrality eigenvector of a network $G$ even if the associated nonbacktracking matrix, $B(0)$, is not irreducible. Indeed, in this case the eigenspace associated to the spectral radius may have dimension greater than one and it might be not possible to select a unique norm-one positive vector inside. But for every $\alpha$ close to zero we can find the $\alpha$-nonbacktracking centrality eigenvector $\mathbf{c}(\alpha)$ of $M_{\alpha}:=B(\alpha)^{t}$ and take $\mathbf{c}=\lim _{\alpha \rightarrow 0^{+}} \mathbf{c}(\alpha)$ (which has been shown to exist) as $\alpha \rightarrow 0^{+}$. Since $\mathbf{c}$ has norm one, is non-negative and belongs to the eigenspace associated to the spectral radius of $M_{0}:=B(0)^{t}$ it is perfectly sound to refer to $\mathbf{c}$ as the nonbacktracking centrality eigenvector of $G$.

The following definition collects this remark.

Definition 4.4. Given a directed network $G$ we call the nonbacktracking edge centrality of $G$ to the vector $\mathbf{c}=\lim _{\alpha \rightarrow 0^{+}} \mathbf{c}(\alpha)$. From this we define the nonbacktracking centrality of a node $i \in G$ as

$$
c_{i}=\sum_{j} c_{i \rightarrow j},
$$

where $c_{i \rightarrow j}$ is the coordinate of $\mathbf{c}$ indexed by the edge $i \rightarrow j$.

Notice again that this definition is consistent with the original one in the case that the nonbacktracking matrix of $G$ is irreducible as was observed in Remark 4.3.

Example 4.5. With this definition the nonbacktracking centrality of the network $G$ in Fig. 5 can be determined. In this example the matrices $B(\alpha)$ and $B(0)$ are as follows, where we are using the lexicographical order for the edges, that is, $(1 \rightarrow 2,2 \rightarrow 1,2 \rightarrow 3,3 \rightarrow 2):$

$$
B(\alpha)=\left(\begin{array}{cccc}
0 & \alpha & 1 & 0 \\
\alpha & 0 & 0 & 0 \\
0 & 0 & 0 & \alpha \\
0 & 1 & \alpha & 0
\end{array}\right) \quad B(0)=\left(\begin{array}{cccc}
0 & 0 & 1 & 0 \\
0 & 0 & 0 & 0 \\
0 & 0 & 0 & 0 \\
0 & 1 & 0 & 0
\end{array}\right)
$$

and the networks corresponding to $B(\alpha)$ and $B(0)$ are those in Figs. 6 and 7 respectively.

We get, through direct computation, that the spectral radius $\rho_{\alpha}=\rho\left(B(\alpha)^{t}\right)=\sqrt{\alpha^{2}+\alpha}$ and that $\mathbf{v}(\alpha)=(\alpha$, $\left.\sqrt{\alpha^{2}+\alpha}, \sqrt{\alpha^{2}+\alpha}, \alpha\right)$ is a positive eigenvector of $B(\alpha)^{t}$ associated to $\rho_{\alpha}$. Therefore

$$
\mathbf{c}(\alpha):=\frac{\mathbf{v}(\alpha)}{\|\mathbf{v}(\alpha)\|_{1}}
$$



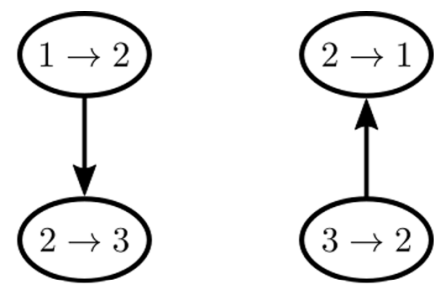

Fig. 7. The network associated to $B(0)$.

is the Perron vector of $B(\alpha)^{t}$. It is easy to prove that

$$
\mathbf{c}=\lim _{\alpha \rightarrow 0^{+}} \mathbf{c}(\alpha)=\left(0, \frac{1}{2}, \frac{1}{2}, 0\right)
$$

and this vector is what we have defined as the nonbacktracking edge centrality of $G$. Note that in this case $\rho(B(0))=0$ and the associated eigenspace $V_{0}=\{(0, \mu, v, 0)\}_{\mu, v \in \mathbb{R}}$ has dimension 2 .

Finally, for the nonbacktracking centrality of the nodes of $G$, we have

$$
c_{1}=c_{1 \rightarrow 2}=0, \quad c_{2}=c_{2 \rightarrow 1}+c_{2 \rightarrow 3}=1, \quad c_{3}=c_{3 \rightarrow 2}=0 .
$$

Remark 4.6. Next we point out how Theorem 4.1 can be used to study the limit case of other spectral centrality measures. An example is the PageRank of $G$ [26], indexed by the damping factor $q$, which is defined as the Perron eigenvector of the matrix

$$
R_{q}=q P^{t}+(1-q) N^{t}=P^{t}+(1-q)\left(N^{t}-P^{t}\right),
$$

where $P$ is obtained by normalization of each row of the adjacency matrix $A$ and $N$ is a personalization matrix (which can be seen as an extension of personalization vectors). If we set $\alpha=1-q, M_{0}=P^{t}$ and $M=N^{t}-P^{t}$ we get $M_{\alpha}=M_{0}+\alpha M=R_{q}$. Therefore Theorem 4.1 proves the convergence of the PageRank vector when $q \rightarrow 1^{-}$. This result has been shown by Boldi et al. in Section 5 of [10] for the case of a matrix $N$ constructed from a personalization vector. Our theorem provides an alternative proof and generalizes the result to the case of an arbitrary personalization matrix. Note that the limit cases of eigenvector-type centralities have been studied in the literature (see, for example $[9,16]$ ) and Theorem 4.1 proves the existence of such limit cases.

\section{Acknowledgments}

This work has been partially supported by projects MTM2014-59906 MTM2014-52470-P (Spanish Ministry), MTM201784194-P (AEI/FEDER, Spain, UE) and the grant URJC-Grupo de Excelencia Investigadora GARECOM, Spain (2014-2017).

\section{References}

[1] S. Boccaletti, G. Bianconi, R. Criado, C.I. Del Genio, J. Gómez-Gardenes, M. Romance, I. Sendina-Nadal, Z. Wang, M. Zanin, The structure and dynamics of multilayer networks, Phys. Rep. 544 (1) (2014) 1-122.

[2] S. Boccaletti, V. Latora, Y. Moreno, M. Chavez, D.-U. Hwang, Complex networks: Structure and dynamics, Phys. Rep. 424 (4) (2006) 175-308.

[3] M. De Domenico, A. Solé-Ribalta, E. Cozzo, M. Kivelä, Y. Moreno, M.A. Porter, S. Gómez, A. Arenas, Mathematical formulation of multilayer networks, Phys. Rev. X 3 (4) (2013) 041022.

[4] E. Estrada, Communicability in temporal networks, Phys. Rev. E 88 (4) (2013) 042811.

[5] P. Holme, J. Saramäki, Temporal networks, Phys. Rep. 519 (3) (2012) 97-125.

[6] H. Kim, J. Tang, R. Anderson, C. Mascolo, Centrality prediction in dynamic human contact networks, Comput. Netw. 56 (3) (2012) 983-996.

[7] M. Kivelä, A. Arenas, M. Barthelemy, J.P. Gleeson, Y. Moreno, M.A. Porter, Multilayer networks, J. Complex Netw. 2 (3) (2014) $203-271$.

[8] M. Newman, Networks: An Introduction, Oxford University Press, 2010.

[9] M. Benzi, C. Klymko, On the limiting behavior of parameter-dependent network centrality measures, SIAM J. Matrix Anal. Appl. 36 (2) (2015) 686-706.

[10] P. Boldi, M. Santini, S. Vigna, PageRank: functional dependencies, ACM Trans. Inf. Syst. (TOIS) 27 (4) (2009) 19.

[11] L. Solá, M. Romance, R. Criado, J. Flores, A. García del Amo, S. Boccaletti, Eigenvector centrality of nodes in multiplex networks, Chaos 23 (3) (2013) 033131.

[12] P. Bonacich, Factoring and weighting approaches to status scores and clique identification, J. Math. Sociol. 2 (1) (1972) 113-120.

[13] R. Criado, J. Flores, A. García del Amo, M. Romance, Centralities of a network and its line graph: an analytical comparison by means of their irregularity, Int. J. Comput. Math. 91 (2) (2014) 304-314.

[14] T. Martin, X. Zhang, M. Newman, Localization and centrality in networks, Phys. Rev. E 90 (5) (2014) 052808

[15] R. Pastor-Satorras, C. Castellano, Distinct types of eigenvector localization in networks, Sci. Rep. 6 (1) (2016) 18847.

[16] N. Perra, S. Fortunato, Spectral centrality measures in complex networks, Phys. Rev. E 78 (3) (2008) 036107.

[17] K.-i. Hashimoto, Zeta functions of finite graphs and representations of p-adic groups, in: Automorphic Forms and Geometry of Arithmetic Varieties, Elsevier, 1989, pp. 211-280.

[18] F. Krzakala, C. Moore, E. Mossel, J. Neeman, A. Sly, L. Zdeborová, P. Zhang, Spectral redemption in clustering sparse networks, Proc. Natl. Acad. Sci. 110 (52) (2013) 20935-20940. 
[19] C.D. Meyer, Matrix Analysis and Applied Linear Algebra, Vol. 2, Siam, 2000.

[20] R. Criado, J. Flores, A.G. del Amo, M. Romance, Analytical relationships between metric and centrality measures of a network and its dual, J. Comput. Appl. Math. 235 (7) (2011) 1775-1780.

[21] R. Criado, J. Flores, A. García del Amo, M. Romance, Structural properties of the line-graphs associated to directed networks, Netw. Heterogen. Media 7 (3) (2012) 373-384.

[22] J. Xu, Topological Structure and Analysis of Interconnection Networks, Vol. 7, Springer Science \& Business Media, 2013.

[23] G. Harris, C. Martin, The roots of a polynomial vary continuously as a function of the coefficients, Proc. Amer. Math. Soc. (1987) 390-392.

[24] T. Kato, Perturbation Theory for Linear Operators, Vol. 132, Springer Science \& Business Media, 1995.

[25] K. Knopp, Theory of Functions, Part II, Dover Publications, New York, 1947.

[26] S. Brin, L. Page, Reprint of: The anatomy of a large-scale hypertextual web search engine, Comput. Netw. 56 (18) (2012) $3825-3833$. 